\title{
Crystal structure of pentachloro(triphenylphosphinesulfide)niobium(V), $\mathrm{NbCl}_{5}\left(\mathbf{P h}_{3} \mathbf{P S}\right)$
}

\author{
K. Stumpf ${ }^{1}$, R. Blachnik ${ }^{*, I}$, G. Roth ${ }^{\mathrm{II}}$ and G. Kastner ${ }^{\mathrm{I}}$

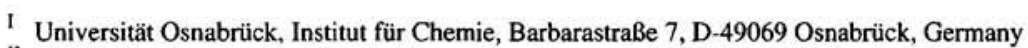 \\ II RWTH Aachen, Institut für Kristallographie, Jägerstraße 17-19, D-52056 Aachen, Germany
}

Received April 11, 2000, CCDC-No. $1267 / 442$

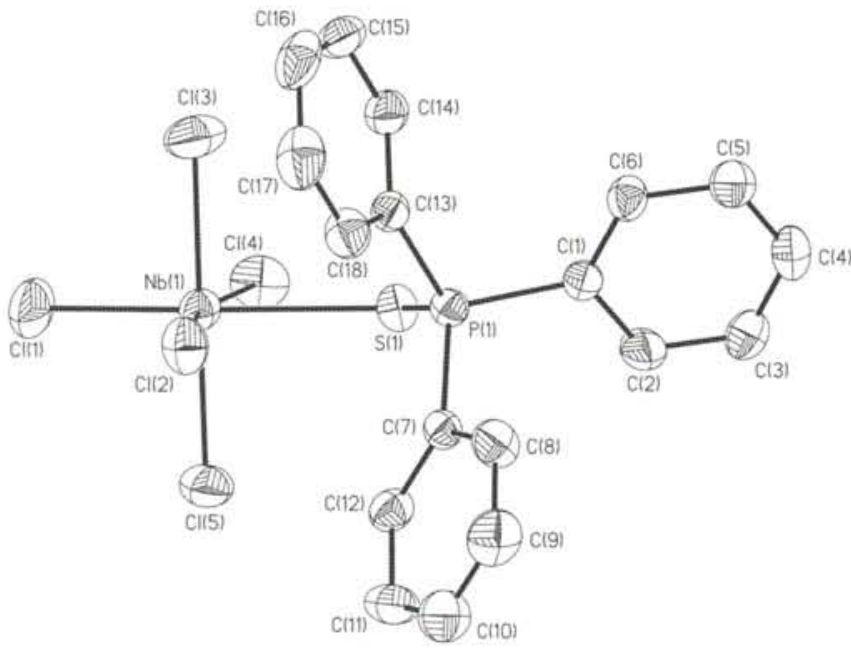

Abstract

$\mathrm{C}_{18} \mathrm{H}_{15} \mathrm{Cl}_{5} \mathrm{NbPS}$, triclinic, $P \overline{\mathrm{l}}$ (No. 2), $a=9.944(2) \AA$,

$b=10.179(2) \AA, c=11.893(2) \AA, \alpha=88.9(8)^{\circ}, \beta=70.25(9)^{\circ}$, $\gamma=72.96(9)^{\circ}, V=1079.0 \AA^{3}, Z=2, R_{\mathrm{gt}}(F)=0.029$,

$w R_{\text {ref }}\left(F^{2}\right)=0.056, T=293 \mathrm{~K}$.

\section{Source of material}

Red crystals of $\mathrm{NbCl}_{5}\left(\mathrm{Ph}_{3} \mathrm{PS}\right)$ were obtained from mixing solutions of niobium pentachloride and triphenylphosphinesulfide in dichloromethane in equimolar ratios. $\mathrm{NbCl}_{5}\left(\mathrm{Ph}_{3} \mathrm{PS}\right)$ is sensitive towards moisture.

\section{Discussion}

The neutral complex consists of approximately regular octahedra centred by niobium atoms. $\mathrm{Nb}-\mathrm{Cl}$ bond lengths are in the range $2.29 \AA-2.35 \AA$, in good agreement with values of $2.25 \AA$ and $2.35 \AA$ in $\mathrm{NbCl}_{5}\left(\mathrm{POCl}_{3}\right)[1]$ and show the usual trans-effect. $\mathrm{Nb}-\mathrm{S}$ length in $\mathrm{NbCl}_{5}\left(\mathrm{Ph}_{3} \mathrm{PS}\right)\left(d_{\mathrm{Nb}-\mathrm{S}}=2.62 \AA\right)$ is close to that in $\mathrm{NbSCl}_{3}\left(\mathrm{Ph}_{3} \mathrm{PS}\right)\left(d_{\mathrm{Nb}}-\mathrm{s}=2.58 \AA\right)[2]$.

Table 1. Data collection and handling.

Crystal:

Wavelength:

$\mu$ :

Diffractometer, scan mode:

$2 \theta_{\max }$ :

$N(h k l)_{\text {measured, }} N(h k l)_{\text {unique: }}$ Criterion for $I_{\mathrm{obs}}, N(h k l)_{\mathrm{gl}}$ :

$N$ (param) refined: Programs:

\author{
red needle, size $0.2 \times 0.2 \times 0.3 \mathrm{~mm}$ \\ Mo $K_{\alpha}$ radiation $(0.71069 \AA$ \\ $13.49 \mathrm{~cm}^{-1}$ \\ Stoe-IPDS, 159 exposures, $\Delta \varphi=1.7^{\circ}$ \\ $56.36^{\circ}$ \\ 13833,4780 \\ $I_{\text {obs }}>2 \sigma\left(I_{\text {obs }}\right), 3085$ \\ 226 \\ SHELXS-97 [3], SHELXL-97 [4]
}

Table 2. Atomic coordinates and displacement parameters (in $\AA^{2}$ ).

\begin{tabular}{llllll}
\hline Atom & Site & $x$ & $y$ & $z$ & $U_{\text {iso }}$ \\
\hline $\mathrm{H}(2)$ & $2 i$ & 0.3160 & 0.7639 & 0.7184 & 0.051 \\
$\mathrm{H}(3)$ & $2 i$ & 0.1016 & 0.9451 & 0.8074 & 0.055 \\
$\mathrm{H}(4)$ & $2 i$ & 0.0901 & 1.0927 & 0.9571 & 0.047 \\
$\mathrm{C}(5)$ & $2 i$ & $0.2950(3)$ & $0.9973(3)$ & $0.9616(3)$ & $0.0401(7)$ \\
$\mathrm{H}(5)$ & $2 i$ & 0.2889 & 1.0555 & 1.0237 & 0.048 \\
$\mathrm{H}(6)$ & $2 i$ & 0.5057 & 0.8748 & 0.9342 & 0.041 \\
$\mathrm{H}(8)$ & $2 i$ & 0.6506 & 0.8981 & 0.6234 & 0.051 \\
$\mathrm{H}(9)$ & $2 i$ & 0.7123 & 0.9578 & 0.4275 & 0.062 \\
$\mathrm{C}(10)$ & $2 i$ & $0.7199(4)$ & $0.7803(3)$ & $0.3547(3)$ & $0.0494(8)$ \\
$\mathrm{H}(10)$ & $2 i$ & 0.7420 & 0.8062 & 0.2767 & 0.059 \\
$\mathrm{H}(11)$ & $2 i$ & 0.7210 & 0.5892 & 0.3136 & 0.056 \\
$\mathrm{H}(12)$ & $2 i$ & 0.6623 & 0.5251 & 0.5087 & 0.042 \\
$\mathrm{H}(14)$ & $2 i$ & 0.6357 & 0.6071 & 0.9625 & 0.046 \\
$\mathrm{H}(15)$ & $2 i$ & 0.8172 & 0.6057 & 1.0446 & 0.061 \\
$\mathrm{H}(16)$ & $2 i$ & 1.0332 & 0.6527 & 0.9313 & 0.068 \\
$\mathrm{H}(17)$ & $2 i$ & 1.0702 & 0.7046 & 0.7358 & 0.065 \\
$\mathrm{H}(18)$ & $2 i$ & 0.8876 & 0.7132 & 0.6543 & 0.050 \\
\hline & & & & & \\
\hline
\end{tabular}

Table 3. Atomic coordinates and displacement parameters (in $\AA^{2}$ ).

\begin{tabular}{llllllllllr}
\hline Atom & Site & $x$ & $y$ & $z$ & $U_{11}$ & $U_{22}$ & $U_{33}$ & \multicolumn{1}{c}{$U_{12}$} & $U_{13}$ & $U_{23}$ \\
\hline $\mathrm{Nb}(1)$ & $2 i$ & $0.75872(3)$ & $0.25522(2)$ & $0.69951(3)$ & $0.0361(2)$ & $0.0264(1)$ & $0.0338(2)$ & $-0.0095(1)$ & $-0.0145(1)$ & $0.0047(1)$ \\
$\mathrm{Cl}(1)$ & $2 i$ & $0.9214(1)$ & $0.03484(7)$ & $0.65677(9)$ & $0.0691(6)$ & $0.0328(4)$ & $0.0748(7)$ & $0.0040(4)$ & $-0.0357(6)$ & $-0.0011(4)$ \\
$\mathrm{Cl}(3)$ & $2 i$ & $0.8063(1)$ & $0.27998(8)$ & $0.87496(8)$ & $0.0817(7)$ & $0.0505(4)$ & $0.0496(6)$ & $-0.0241(4)$ & $-0.0417(5)$ & $0.0110(4)$ \\
$\mathrm{Cl}(2)$ & $2 i$ & $0.91983(9)$ & $0.37788(7)$ & $0.60401(8)$ & $0.0337(4)$ & $0.0421(4)$ & $0.0521(5)$ & $-0.0137(3)$ & $-0.0058(4)$ & $0.0064(3)$ \\
\hline
\end{tabular}

\footnotetext{
* Correspondence author (e-mail: rblachni@rz.uni-osnabrueck.de)
} 
Table 3. Continued.

\begin{tabular}{|c|c|c|c|c|c|c|c|c|c|c|}
\hline Atom & Site & $x$ & $y$ & $z$ & $U_{11}$ & $U_{22}$ & $U_{33}$ & $U_{12}$ & $U_{13}$ & $U_{23}$ \\
\hline $\mathrm{Cl}(4)$ & $2 i$ & $0.5479(1)$ & $0.18552(7)$ & $0.79963(8)$ & $0.0561(5)$ & $0.0500(4)$ & $0.0485(5)$ & $-0.0316(4)$ & $-0.0160(5)$ & $0.0127(4)$ \\
\hline $\mathrm{Cl}(5)$ & $2 i$ & $0.7129(1)$ & $0.23504(7)$ & $0.51956(7)$ & $0.0648(6)$ & $0.0461(4)$ & $0.0390(5)$ & $-0.0122(4)$ & $-0.0253(5)$ & $-0.0023(3)$ \\
\hline$S(1)$ & $2 i$ & $0.53908(8)$ & $0.48707(6)$ & $0.76366(7)$ & $0.0299(4)$ & $0.0286(3)$ & $0.0371(4)$ & $-0.0110(3)$ & $-0.0078(4)$ & $0.0023(3)$ \\
\hline $\mathbf{P}(1)$ & $2 i$ & $0.60057(8)$ & $0.66202(6)$ & $0.73850(6)$ & $0.0273(4)$ & $0.0265(3)$ & $0.0260(4)$ & $-0.0084(3)$ & $-0.0093(4)$ & $0.0011(3)$ \\
\hline$C(1)$ & $2 i$ & $0.4336(3)$ & $0.8011(2)$ & $0.8173(2)$ & $0.027(2)$ & $0.029(1)$ & $0.027(2)$ & $-0.010(1)$ & $-0.007(1)$ & $0.006(1)$ \\
\hline $\mathrm{C}(2)$ & $2 i$ & $0.3113(3)$ & $0.8227(3)$ & $0.7795(3)$ & $0.039(2)$ & $0.052(2)$ & $0.038(2)$ & $-0.008(1)$ & $-0.019(2)$ & $-0.009(1)$ \\
\hline$C(3)$ & $2 i$ & $0.1834(3)$ & $0.9310(3)$ & $0.8325(3)$ & $0.031(2)$ & $0.054(2)$ & $0.049(2)$ & $-0.005(1)$ & $-0.019(2)$ & $0.002(2)$ \\
\hline$C(4)$ & $2 i$ & $0.1761(3)$ & $1.0187(3)$ & $0.9226(3)$ & $0.031(2)$ & $0.030(1)$ & $0.047(2)$ & $-0.005(1)$ & $-0.004(2)$ & $0.005(1)$ \\
\hline$C(6)$ & $2 i$ & $0.4245(3)$ & $0.8886(2)$ & $0.9084(3)$ & $0.032(2)$ & $0.031(1)$ & $0.042(2)$ & $-0.007(1)$ & $-0.017(2)$ & $-0.001(1)$ \\
\hline$C(7)$ & $2 i$ & $0.6534(3)$ & $0.7038(2)$ & $0.5853(2)$ & $0.024(2)$ & $0.031(1)$ & $0.026(2)$ & $-0.007(1)$ & $-0.005(1)$ & $0.003(1)$ \\
\hline $\mathrm{C}(8)$ & $2 i$ & $0.6659(4)$ & $0.8351(3)$ & $0.5613(3)$ & $0.050(2)$ & $0.035(1)$ & $0.039(2)$ & $-0.014(1)$ & $-0.011(2)$ & $0.004(1)$ \\
\hline$C(9)$ & $2 i$ & $0.7013(4)$ & $0.8711(3)$ & $0.4444(3)$ & $0.058(2)$ & $0.043(2)$ & $0.053(2)$ & $-0.022(2)$ & $-0.015(2)$ & $0.022(2)$ \\
\hline$C(11)$ & $2 i$ & $0.7069(4)$ & $0.6506(3)$ & $0.3764(3)$ & $0.054(2)$ & $0.057(2)$ & $0.031(2)$ & $-0.018(2)$ & $-0.017(2)$ & $0.002(1)$ \\
\hline$C(12)$ & $2 i$ & $0.6725(3)$ & $0.6122(3)$ & $0.4929(3)$ & $0.040(2)$ & $0.031(1)$ & $0.038(2)$ & $-0.011(1)$ & $-0.019(2)$ & $0.007(1)$ \\
\hline$C(13)$ & $2 i$ & $0.7436(3)$ & $0.6615(2)$ & $0.7996(3)$ & $0.030(2)$ & $0.026(1)$ & $0.034(2)$ & $-0.006(1)$ & $-0.015(2)$ & $-0.002(1)$ \\
\hline$C(14)$ & $2 i$ & $0.7222(4)$ & $0.6280(2)$ & $0.9171(3)$ & $0.045(2)$ & $0.030(1)$ & $0.040(2)$ & $-0.007(1)$ & $-0.019(2)$ & $0.002(1)$ \\
\hline$C(15)$ & $2 i$ & $0.8314(4)$ & $0.6263(3)$ & $0.9657(3)$ & $0.074(3)$ & $0.035(2)$ & $0.052(2)$ & $-0.002(2)$ & $-0.044(2)$ & $-0.001(1)$ \\
\hline$C(16)$ & $2 i$ & $0.9603(4)$ & $0.6547(3)$ & $0.8980(4)$ & $0.055(2)$ & $0.038(2)$ & $0.090(3)$ & $-0.002(2)$ & $-0.051(3)$ & $-0.009(2)$ \\
\hline$C(17)$ & $2 i$ & $0.9824(4)$ & $0.6863(3)$ & $0.7815(4)$ & $0.037(2)$ & $0.049(2)$ & $0.081(3)$ & $-0.013(2)$ & $-0.025(2)$ & $-0.009(2)$ \\
\hline $\mathrm{C}(18)$ & $2 i$ & $0.8734(3)$ & $0.6906(3)$ & $0.7328(3)$ & $0.035(2)$ & $0.043(2)$ & $0.048(2)$ & $-0.012(1)$ & $-0.015(2)$ & $-0.002(1)$ \\
\hline
\end{tabular}

Acknowledgments. The authors thank the Deutsche Forschungsgemeinschaft (SFB 225) and the Fonds der Chemie for their support.

\section{References}

1. Bränden, C.-I.; Lindqvist, I.: The Crystal Structures of the Adducts $\mathrm{SbCl}_{5} \cdot\left(\mathrm{CH}_{3}\right)_{3} \mathrm{PO}$ and $\mathrm{NbCl}_{5} \cdot \mathrm{POCl}_{3}$. Acta Chem. Scand. 17 (1963) 353-361.
2. Drew, M. G. B.; Hobson, R. J.: Preparation, Crystal and Molecular Structure of Trichlorosulphido(triphenylphosphine sulphide)niobium(V). Inorg. Chim. Acta 72 (1983) 233-237.

3. Sheldrick, G. M.: SHELXS-97. Program for the Solution of Crystal Structures. University of Göttingen, Germany 1986.

4. Sheldrick, G. M.: SHELXL-97. Program for the Refinement of Crystal Structures. University of Göttingen, Germany 1997. 